\title{
Hubert Gabrisch
}

\section{Währung ohne Souverän: Zur Ursache und Überwindung der Euro- Krise ${ }^{1}$}

In der Europäischen Union wird seit 2010 nach Wegen der Reform ihres institutionellen Gefüges gesucht, um das Weiterbestehen des Euro-Raums zu sichern. Viele Beobachter, aber auch Investoren haben jedoch angesichts der Vielzahl der offiziellen Reformvorschläge den roten Faden verloren und vermissen ein Konzept, welches über die Erfordernisse des Tages hinausreicht. Dies hat negative Auswirkungen auf die Wirtschaft des Euro-Raums. Hohe Risiken in den Finanzsektoren lähmen die notwendige Kreditvergabe an den privaten Unternehmenssektor in den Krisenländern, und die bisherigen und erwarteten Budgetkürzungen in Schuldnerländern haben 2012 wiederum eine Rezession ausgelöst, die zweite nach der vom Jahr 2009 im gesamten Euro-Raum, den prophezeiten und gefürchteten Double-Dip.

Ziel dieses Beitrags ist es, die Risiken der gegenwärtigen Reformpolitik aufzuzeigen. Es geht um den Fiskalpakt vom März 2012, der neben dem Europäischen Stabilitätsmechanismus (ESM) die bisher wichtigste Reform ist und auf eine Konsolidierung der öffentlichen Finanzen abzielt, deren Krise als Ursache der Euro-Krise gesehen wird. Was indes als fiskalische Krise verstanden wird, ist in Wirklichkeit eine Zahlungsbilanzkrise. Dieser Irrtum ist an anderer Stelle ausführlich behandelt worden, ${ }^{2}$ sodass darauf nicht weiter eingegangen wird.

Zur Beurteilung des Fiskalpakts reicht eine technische Analyse seiner Wirkungen auf Einkommen und Beschäftigung nicht aus. Deshalb wird hier im Unterschied zu anderen kritischen Debattenbeiträgen eine theoretische Fokussierung geboten. Mein zentrales Argument lautet, dass eine Währung nur so stark ist wie der Staat oder auch der Souverän, der hinter ihr steht. Eine derartige Autorität fehlt dem Euro. Was das Argument konkret bedeutet, ist Gegenstand des ersten Kapitels, in welchem ich mich kritisch mit der Theorie des optimalen Währungsraums befasse, die die Argumente für eine Währungsunion, wie sie besteht und wie sie reformiert werden sollte, liefert. Eine Schlussfolgerung dieser Kritik lautet, dass in einer Währungsunion wegen des fehlenden Souveräns eine dauerhafte Spaltung in Gläubiger- und Schuldnerländer auftritt und nachfolgende fiskalische Restriktionen in den Schuldnerländern zu deflationären Tendenzen mit steigender Arbeitslosigkeit im gesamten Währungsgebiet führen. Diese Schlussfolgerung wird im zweiten Kapitel anhand des Fiskalpakts untersucht. Kern ist eine Schätzung der Auswirkungen auf das

1 Ich danke zwei anonymen Gutachtern sowie Karl Betz (Meschede), Kazimierz Laski (Wien) und Achim Truger (Berlin) für hilfreiche Kommentare.

2 Siehe beispielsweise Illing et al. 2012.

Leviathan, 41. Jg., 1/2013, S. 121/ro 143 
aggregierte Einkommen des Euro-Raums. ${ }^{3}$ Im Unterschied zu den meisten Debattenbeiträgen komme ich zu einer negativen Beurteilung des Fiskalpakts hinsichtlich seines Beitrags zu einer Überwindung der institutionellen Schwäche des EuroRaums. Das dritte Kapitel befasst sich mit Reformvorhaben, wie die Währungsunion in einen »souveränen Währungsraum « überführt werden kann. Im letzten Kapitel findet sich eine Zusammenfassung. In einem Anhang lege ich die methodischen Grundlagen meiner Schätzungen dar.

\section{Theorie der Währungsunion}

\subsection{Warum Währungsunionen scheitern}

Souveräner Staat und nationale Währung bilden einen »souveränen Währungsraum «. Er ist der Normalfall in der Weltwirtschaft. Dagegen kombiniert eine Währungsunion mehrere Nationalstaaten ohne Schaffung eines souveränen Gesamtstaates. Somit ist die Geldpolitik zentralisiert und die Fiskalpolitik auf nationaler Ebene dezentralisiert. Die Wirtschaftsgeschichte, insbesondere die jüngere, zeigt indes, dass dem Sonderfall einer Währungsunion in der Geschichte nie eine lange Lebenszeit bemessen war: Entweder sie zerfielen oder ihre Mitglieder vereinigten sich zu einem Staat. Heute gibt es noch vier Währungsunionen, von denen nur eine - die Europäische Währungsunion (EWU) - aus entwickelten Industriestaaten besteht, die anderen dagegen aus Entwicklungsländern. Im europäischen Einigungsprozess wurde ursprünglich im Rahmen einer weitergehenden politischen Union eine Zentralisierung der Fiskalpolitik in Betracht gezogen (Werner-Plan 1970). Dieser Plan wurde aber bei der Konstruktion der EU (Maastricht-Vertrag 1992) fallen gelassen. Neben der EWU gibt es derzeit noch die beiden CFA-Zonen in Westafrika, gestützt durch das französische Finanzministerium, und die Eastern Caribbean Currency Union (ECCU) mit einer Zentralbank, aber ebenfalls ohne politische Union. Die Geschichte der Währungsunionen ist auch eine des Scheiterns: Die Lateinische Münzunion hielt von 1865 bis 1914/1926, ähnlich auch die Skandinavische Währungsunion (1872-1924). Die Rubel-Zone zerbrach 1993 relativ schnell nach dem Zusammenbruch der Sowjetunion und dem Entstehen vieler Nachfolgestaaten. Auch die Tschechisch-Slowakische Währungsunion lebte nach der Auflösung des gemeinsamen Staates nur vom 31. Dezember 1992 bis zum 7. Februar 1993. Der Dinar verschwand nach dem gewaltsamen Zerfall Jugoslawiens in den frühen 1990er Jahren. Sicherlich gab es für jedes Beispiel auch unterschiedliche politische und ökonomische Gründe. Aber ein gemeinsamer Faktor ist relativ leicht erkennbar: Währungsunionen fehlt der gemeinsame Staat oder, anders ausgedrückt, eine staatliche Union mit einer zentralen Finanzbehörde, die gemeinsam mit der Zentralbank verantwortlich für die Währung und ihre Stabilität ist. Dabei scheint es so, dass eine

3 In ihren groben Ergebnissen war die Analyse bereits im November 2012 fertig. Seitdem hat die EU-Kommission ihre Prognosen für 2012 und 2013 nach unten revidiert und mit ihrem Armutsbericht im Januar 2013 meine Berechnungen und Befürchtungen bezüglich der Effekte des Fiskalpakts im Nachhinein bestätigt. 
gemeinsame Zentralbank offensichtlich weniger bedeutsam ist als ein gemeinsamer Staat. So besaß die Skandinavische Währungsunion keine gemeinsame Zentralbank, basierte aber zumindest auf der staatlichen Union zwischen Schweden und Norwegen. Ihre Auflösung 1905 bereitete den Weg für das spätere Ende der monetären Union.

Eine stärkeres intuitives Verständnis für das Argument liefern vielleicht Erfolgsgeschichten: Der US-Dollar wurde zur führenden Reservewährung, nachdem die lockere Konföderation der ehemaligen amerikanischen Kolonien Englands - institutionell durchaus vergleichbar mit der EU - nach dem gewonnenen Unabhängigkeitskrieg 1787 in eine Union mit Zentralregierung überführt wurde. Dabei hat es nicht geschadet, dass die Neu-England-Staaten, die Mittelatlantik-Staaten und der Süden eine durchwegs unterschiedliche "Stabilitätskultur « mit unterschiedlichen öffentlichen Verschuldungsständen aufwiesen. ${ }^{4}$ Es scheint, verfolgt man die Literatur über die jungen Vereinigten Staaten, eher so, dass gerade diese Unterschiede eine starke fiskalische Zentralregierung erzwangen, wenn nicht andernfalls die neue Union schon nach wenigen Jahren scheitern sollte. Die Durchsetzung des Dollar als gemeinsame Währung folgte den Höhen und Tiefen der Unionsbildung und endete erst mit Gründung des Federal Reserve Systems 1913. Ein anderes Beispiel liefert die deutsch-deutsche Währungsunion: Die Deutsche Mark (DM) verlor ihre Stellung als Reservewährung nicht, als sie im Juli 1990 in der DDR eingeführt wurde, denn die staatliche Vereinigung erfolgte im Oktober 1990, drei Monate nach der Einführung der DM in der DDR. Die Belgisch-Luxemburgische Währungsunion war eine Erfolgsgeschichte, weil Luxemburg nur ein kleiner Appendix war und belgische Zentralbank und Finanzministerium leicht die gemeinsame Währung decken konnten. Aus all dem kann auch positiv geschlossen werden: Die notwendige Bedingung für eine souveräne Währung ist ein Staat, der mit seiner Steuerkraft hinter dieser Währung steht. In der europäischen Währungsunion lehnen jedoch die Staaten mit der größten Steuerkraft - insbesondere Deutschland - eine derartige Übernahme von Verantwortung ab - ganz ähnlich wie es 1790 zunächst die Südstaaten gegenüber den Nordstaaten der USA taten, um dann letztlich doch umzuschwenken. ${ }^{5}$

\subsection{Kritik der Theorie des optimalen Währungsraums}

Dass in Europa eine gemeinsame Währung ohne einen Souverän eingeführt wurde, verweist auf eine Denkweise, die Staat und Märkte separiert. Diese Denkweise zeichnet viele Theorien aus, die unter dem Begriff des Marktfundamentalismus subsumiert werden können. Dazu gehört auch die Theorie des optimalen Währungsraums (OWR), die das Denken vieler Ökonomen beeinflusst hat - sowohl der Befürworter wie auch der Kritiker der Einführung einer gemeinsamen Währung in

4 Was auch für die heutigen USA gilt, die nach wie vor nicht als optimaler Währungsraum gelten.

5 Lesenswert ist hierzu J.J. Ellis' faktenreicher Essay »Das Dinner « in seinem Buch über die Gründergeneration der USA, der vielleicht auch das Interesse unserer Politiker finden könnte; Ellis 2002.

Leviathan, 41. Jg., 1/2013 
Europa. ${ }^{6}$ Die Theorie des OWR wurde in den 1960er Jahren entwickelt, um die Frage nach der notwendigen Anzahl von Währungen in der Weltwirtschaft und den Voraussetzungen für den Zusammenschluss regionaler Währungen zu einer einheitlichen Währung zu beantworten. Sie ist insofern keine Theorie der Währungsunion; ihre Kriterien können auf Nationalstaaten mit eigener Währung und mehreren Regionen genauso angewendet werden wie auf die währungspolitische Zusammenarbeit zwischen Staaten oder eben auf eine Währungsunion. Die traditionellen Kriterien für einen optimalen Währungsraum sind flexible Arbeitsmärkte ${ }^{7}$, offene Gütermärkte ${ }^{8}$ und diversifizierte Produktionsstrukturen ${ }^{9}$. Krugman ${ }^{10}$ sowie Bayoumi und Eichengreen ${ }^{11}$ fanden, dass diese Kriterien in Europa nicht erfüllt sein würden, und waren daher skeptisch gegenüber der Einführung einer Gemeinschaftswährung. Frankel und Rose ${ }^{12}$ und andere argumentierten dagegen, dass der durch die Gemeinschaftswährung ausgelöste Anpassungszwang die institutionellen und politischen Optimalitätsbedingungen ex-post herbeiführen würde.

De Grauwes Kritik postulierte, dass ein Währungsraum nur dann optimal sein könne, wenn ein System fiskalischer Transfers einen Ausgleich asymmetrischer Schocks sichern würde, der von den Märkten allein nicht erreicht werden könnte. ${ }^{13}$ Damit verweist er implizit auf eine auffallende Eigenschaft der Theorie des OWR, nämlich die Abwesenheit eines Staates in seiner Funktion als fiskalpolitischer und hoheitsrechtlicher Akteur; letzteres betrifft die Durchsetzung des Gebrauchs eines bestimmten Geldes. Staatliches Handeln wäre also nach de Grauwe nur dann gefragt, wenn der Währungsraum nicht optimal funktioniert. Dann ist aber auch zu schlussfolgern: Wenn diese Optimalität besteht oder über eine Reform der Märkte erreicht werden kann, werden fiskalische Transfers überflüssig. Wenn aber staatliches Handeln überflüssig wird, wie kommt dann das Geld in die Wirtschaft und wer sorgt dann für dessen Gebrauch?

Goodhart ${ }^{14}$ argumentierte, dass die Theorie des OWR die räumliche Ausprägung einer Theorie sei, mit der die Mehrzahl der Ökonomen gewöhnlich die Natur und die Entwicklung des Geldes erklären - den sogenannten Metallismus. Dieser Begriff geht wohl auf Schumpeter ${ }^{15}$ zurück; die Theorie selbst hat weiter reichende Wurzeln. Dem Metallismus zufolge ist Geld ein Gut, das auch privat produziert werden

6 Vgl. dazu die führenden Lehrbücher zur europäischen Integration: de Grauwe 1994; Baldwin, Wyplosz 2009, Kapitel 11; Wagener, Eger 2009, Kapitel 14.6.

7 Mundell 1961.

8 McKinnon 1963.

9 Kenen 1969.

10 Krugman 1991.

11 Bayoumi, Eichengreen 1993.

12 Frankel, Rose 1998.

13 Siehe de Grauwe 1994; ähnlich auch Kenen 1969.

14 Goodhart 1998.

15 Schumpeter 1994 [1954], S. 288. 
kann und die Eigenschaft eines Numeraire in Markttransaktionen besitzt. Somit entsteht Geld aus marktwirtschaftlichen Transaktionen und geht ihnen nicht voraus. Jede Geldform und ihr Gebrauch können sich entwickeln und vollständig unabhängig sein von der Existenz eines Staates. Baldwin und Wyplosz begründen in ihrem Lehrbuch zur europäischen Integration explizit die Einführung einer gemeinsamen Währung damit, dass Geld in der Lage sei, die Transaktionskosten von Bartergeschäften zu senken. ${ }^{16}$ Es ist dann nur ein kleiner Schritt zu der Behauptung, dass Geld für marktwirtschaftliche Transaktionen besser ohne staatliche Eingriffe funktioniert. Auf die Praxis der Gestaltung einer Währungsunion angewendet, liegt es dann nahe, den monetären vom fiskalischen Bereich zu trennen und die Währungsunion so zu konstruieren, dass die Zentralbank ausschließlich für die Preisstabilität zuständig ist, während die dezentrale Fiskalpolitik für ein ausgeglichenes Budget zu sorgen hat. Auf diese Weise soll gesichert werden, dass staatliche Einflüsse die Geldfunktionen in der Marktwirtschaft nicht stören.

Die Annahme, dass sich das Geld notwendig aus den privaten Tauschprozessen heraus entwickelt hätte, ist vor dem Hintergrund der uns bekannten Geschichte des Geldes zumindest zweifelhaft. Diese lässt uns eher vermuten, dass Geld bereits verwendet wurde, als Marktwirtschaften überhaupt noch nicht existierten. Historisch belegte Beispiele dafür sind das angelsächsische wergeld ${ }^{17}$, das bei der Ermordung eines Menschen als Sühne und Kompensation diente (erwähnt in der Lex Frisionum, dem »Gesetz der Friesen « aus dem 8. Jahrhundert), oder Geldzahlungen zur Abwehr von Blutrache. ${ }^{18}$ Goodhart ${ }^{19}$ argumentierte auch, dass Geld aus dem Versuch des Herrschers heraus entstand, die Steuerzahlungen seiner Untertanen effizienter zu machen. Das setzt voraus, dass er die Untertanen dazu zwingen musste, Steuerzahlungen nur in der von ihm herausgegebenen Währung zu tätigen. Tatsächlich wurde bisher noch keine altertümliche Münze mit dem Aufdruck eines privaten Emittenten gefunden. Münzen wurden in Münzpressen geprägt, die sich im Eigentum des Souveräns befanden. In der monetären Sprachwelt verweisen einige Begriffe auf diese Ursprünge des Geldes: Der Begriff des »legal tender " beschreibt den gesetzlichen Zwang, ein bestimmtes Geld zu verwenden; in fast allen Staaten ist die Landeswährung gesetzlich als Zahlungsmittel festgelegt, in einigen Ländern (zum Beispiel Ungarn) sogar namentlich in der Verfassung festgeschrieben. Ein anderer Begriff »Seigniorage - beschreibt das Einkommen des Herrschers (»Seigniors«) aus der Münzprägung, nämlich die Differenz zwischen den Kosten der Produktion und dem nominalen Wert der Münze. Papiergeld wurde erstmals vor 1.000 Jahren in China und vor 600 Jahren in Italien verwendet, allein zum Zwecke der Finanzierung der Kriegskosten des Fürsten. Heute versteht man unter Seigniorage das Zinseinkommen aus der privaten Haltung von Münzen und Banknoten, welches von der Zen-

16 Baldwin, Wyplosz 2009, S. 316.

17 Grierson 1977, S. 19-21.

18 Wie es im heutigen Europa noch in einigen Regionen Albaniens üblich zu sein scheint.

19 Goodhart 1998.

Leviathan, 41. Jg., 1/2013 
tralbank an das Schatzamt des Souveräns (Finanzministerium) abgeführt wird. ${ }^{20}$ Dies macht deutlich, dass die monetären und fiskalischen Funktionen des Staates nicht separierbar sind. Wie auch immer die Unabhängigkeit einer Zentralbank institutionell gestaltet ist, funktional betrachtet kann sie nicht unabhängig vom Souverän agieren. Die Ableitung des Geldes aus dem Hoheitsrecht liegt auch Knapps Staatstheorie des Geldes ${ }^{21}$ oder dem Chartalismus zugrunde. Nach Keynes ist »all civilised money [...] chartalist «. ${ }^{22}$ Moderne Chartalisten ${ }^{23}$ verweisen darauf, dass Papiergeld als Kredit eine soziale Konvention und kein Numeraire-Gut sei.

\subsection{Institutionelle Probleme einer Währungsunion}

Da eine Währungsunion keinen Souverän besitzt, hat sie mit institutionellen Problemen zu kämpfen, denen ein einheitlicher Währungsraum nicht ausgesetzt ist. Ein Problem ist die Spaltung in Gläubiger- und Schuldnerstaaten. Die gemeinsame Währung und die einheitliche Geldpolitik der Zentralbank führen in einer ersten Phase zu einer Konvergenz der Risikoprämien auf mittel- und langfristige Zinssätze, denn die bisher regionalen Finanzmärkte verschmelzen zu einem einheitlichen Finanzmarkt - und dies auch dann, wenn strukturelle und entwicklungsbedingte Unterschiede zwischen den Regionen bestehen bleiben, deren Effekte auf die grenzüberschreitenden Wirtschaftsbeziehungen einer Region im Normalfall ja durch deren eigene Währung gemildert werden. Der wichtigste Grund für die Zinskonvergenz ist der Wegfall des Wechselkursrisikos für die Investoren und Anleger. ${ }^{24}$ Die Differenzen zwischen den einzelnen Staaten in ihren fundamentalen Risikofaktoren (z.B. Institutionen) wie auch Niveaus (z.B. Lohnkosten) verschwinden jedoch nicht, und so löst die Konvergenz der Risikoprämien Kapitalströme aus, die zu anhaltenden Leistungsbilanzdefiziten und zu nicht nachhaltigen Gläubiger-Schuldner-Positionen führen. In dem Moment, in dem die Finanzmärkte die fehlende Tragfähigkeit der Schuldnerpositionen erkennen, zerfällt daher der gemeinsame Finanzmarkt wieder in seine regionalen Bestandteile. Die Zinskonvergenz wird durch eine Phase der Zinsdivergenz abgelöst. Es existiert jedoch keine zentrale fiskalische Autorität, die dem hiermit einhergehenden Stabilitätsverlust der Schuldner etwas entgegensetzen könnte, etwa durch vertikale Transfers von der Zentrale zur Region oder durch horizontale fiskalische Transfers zwischen den Regionen. Ein Beispiel für beides in einem souveränen Währungsraum ist die deutsche Gemeinschaftsaufgabe zur Verbesserung der regionalen Wirtschaftsstruktur, mit der Milliarden von Euro von West- nach Ost-Deutschland transferiert wurden und werden.

20 Im Euroraum sind die nationalen Zentralbanken Eigentümer der EZB, wobei sie allerdings selbst wieder im Eigentum der Nationalstaaten sind.

21 Knapp 1924.

22 Keynes 1935, S. 4.

23 Unter anderem Goodhart 1998; Fontana 2003; Lavoie 2011.

24 Wobei auch das sogenannte »risk-sharing «-Argument zu berücksichtigen ist (Mundell 1973; McKinnon 2002). 
Ein zweites Problem ist der Mangel an zentraler Koordination. Wenn ein externer Schock die fehlende Tragfähigkeit der Gläubiger-Schuldner-Positionen aufgedeckt hat, gibt es in einer Währungsunion keine zentrale Behörde, die die fiskalischen Rettungsaktionen mit den monetären Aktionen der Zentralbank koordinieren kann. In einem souveränen Währungsraum ist diese Behörde gewöhnlich das Finanzministerium. Kommt es zu einer schweren Finanzkrise, übernimmt das Finanzministerium die Führung in der gemeinsamen Abwehr. Ein gutes Beispiel dafür ist die Rettungsaktion für das US-amerikanische Finanzsystem 2008 durch das US-Finanzministerium und die amerikanische Zentralbank (die »Fed «) unter Führung des damaligen Finanzministers Paulson. Dagegen muss in einer Währungsunion die Zentralbank mit dem Europäischen Rat, der zuvor zu einer Abstimmung unter den nationalen Finanzministerien gekommen sein muss, verhandeln. Dies muss in einer Krisenlage, die schnelles Handeln erfordert, faktisch eine Übertragung der Führerschaft auf die Zentralbank bewirken. Das jüngste Beispiel dafür war die einseitige Erklärung der EZB vom 6. September 2012, unbegrenzt Staatsanleihen von Krisenländern mit bis zu dreijähriger Laufzeit anzukaufen, um den Zinssatz zu drücken - vorausgesetzt das Land unterwirft sich einem fiskalischen Reformprogramm. Damit übernimmt die Zentralbank auch die Verantwortung dafür, wie eine fiskalische Krise gemanagt wird und wie die Kosten dafür über die nationalen Budgets auf die Steuerzahler und Wähler verteilt werden - alles Kompetenzen einer souveränen Regierung.

Ein drittes Problem ist die nur begrenzte Fähigkeit einer Zentralbank, als Lender of Last Resort (LOLR) im Falle einer privaten Finanzkrise oder einer Finanzkrise eines Mitgliedstaates aufzutreten. In einem souveränen Währungssystem kann eine Zentralbank nicht zusammenbrechen, weil ihre Verluste Teil des Staatsdefizits sind und das Finanzministerium über Steuern (Kapitalerhöhung) oder den Verzicht auf Seigniorage deckt. Wie Goodhart formulierte: »[...] was hinter den Verbindlichkeiten der Zentralbank steht, ist nicht das Kapital der Zentralbank, sondern die Wirtschaftskraft und die Steuerkraft des Staates « 25 . Fehlt diese Voraussetzung der Wirtschaftskraft und Steuerkraft, kann auch eine Zentralbank zusammenbrechen. ${ }^{26}$

\subsection{Beschränkungen der Fiskalpolitik in einer Währungsunion}

Vor etwa 70 Jahren publizierte Abba P. Lerner sein Werk über funktionale Finanzpolitik:27 "Der zentrale Gedanke ist, dass die Fiskalpolitik der Regierung, ihre Ausgaben und ihre Steuerpolitik, ihre Kreditaufnahme und ihre Kreditrückzahlung, ihre Ausdehnung und ihre Einschränkung der Geldmenge nur mit Blick auf die Ergebnisse dieser Aktionen für die Wirtschaft getätigt werden sollen und keinesfalls im

$25 »[\ldots]$ what stands behind of the liabilities of the CB is not the capital of the CB but the strength and taxing power of the State «; Goodhart 1999, S. 347-348.

26 Ähnlich auch Buiter 2008.

27 In wichtigen Teilen gehören dazu Aufgaben, die im deutschen Sprachgebrauch als Fiskalpolitik bezeichnet werden. Der Begriff »Finanzpolitik « wird im Weiteren beibehalten und umfasst Fiskalpolitik als Teilbereich.

Leviathan, 41. Jg., 1/2013 
Hinblick auf irgendeine herkömmliche Doktrin über die Frage, was gesunde oder ungesunde Politik sei. «28

Vier kommentierende Bemerkungen sind hierzu notwendig: Erstens dient funktionale Finanzpolitik zur Bekämpfung von Arbeitslosigkeit oder Inflation durch Anpassungen der öffentlichen Ausgaben und Einnahmen. Welche Alternative der Staat in jeder dieser Situationen wählen wird, hängt von den Grenzkosten einer Erhöhung der Steuern in Relation zu den Grenzkosten einer Erhöhung der Seigniorage ab. Die Kosten einer Steuererhöhung sind im Allgemeinen in Steuervermeidung der Privaten oder/und in erhöhten Ausgaben für die Steuererhebung zu suchen. Eine Erhöhung der Seigniorage kann dagegen selbst zur Inflation beitragen. Zweitens bezieht sich funktionale Finanzpolitik auf eine souveräne Währung. Lerner hatte die Zentralregierung der Vereinigten Staaten als Herausgeber des US-Dollars im Blick und nicht die einzelnen Bundesstaaten. Funktionale Finanzpolitik schließt damit auch nicht die Verschuldung in fremder Währung ein. "Sound Finance " ist das fiskalpolitische Konzept, das eine Regierung bei überwiegender Fremdwährungsverschuldung berücksichtigen muss. Drittens umfasst Lerners Konzept von Regierung sowohl monetäre wie auch fiskalische Funktionen, wobei die Zentralbank nicht notwendigerweise eine Abteilung der Regierung ist. Das Konzept zielt aber auf eine Koordination beider Funktionen ab; die Unabhängigkeit der Zentralbank ist dann in der Anwendung ihrer Instrumente und nicht in der Setzung ihrer Ziele zu verstehen, was dem Souverän zukäme. ${ }^{29}$ Die Herausgabe von Schatztiteln würde von der Zentralbank durch den Einsatz ihrer geldpolitischen Instrumente zu flankieren sein. Und viertens steht hinter dem Konzept der funktionalen Finanzpolitik die Annahme, dass ein Staat, der vorwiegend in eigener Währung verschuldet ist, nicht Bankrott gehen kann, da er neues Papiergeld für die Rückzahlung der aufgenommenen Kredite emittieren oder die Steuern erhöhen kann oder auch die Zinszahlungen als Seigniorage wieder zurückerhält.

Die allgemeine Schlussfolgerung lautet, dass funktionale Finanzpolitik eine optimale Haushaltspolitik für monetär souveräne Staaten offeriert, die sie unabhängig von Übertreibungen der Finanzmärkte macht. Sie kann zur Stabilität der Finanzmärkte beitragen, indem sie risikoarme Anlagemöglichkeiten bietet. Dagegen wird ein Staat mit dem Beitritt zu einer Währungsunion auf die Stufe einer Provinzregierung herabgestuft, die nur dann eine fiskalische Defizitpolitik betreiben kann, wenn die Finanzmärkte bereit sind, sie zu finanzieren. ${ }^{30}$ Dies ist der Grund für die These, dass für eine »Provinzregierung" - oder ein Euro-Land - Verschuldung in der

28 » The central idea is that government fiscal policy, its spending and taxing, its borrowing and repayment of loans, its issue of new money and its withdrawal of money, shall all be undertaken with an eye only on the results of these actions on the economy and not to any established traditional doctrine about what is sound or unsound «; Lerner 1943, S. 39.

29 Bibow macht hier eine Referenz zu Keynes Ablehnung eines Versuchs der britischen Labour-Party aus dem Jahre 1932, parlamentarischen Einfluss auf die Geldpolitik zu nehmen; siehe Bibow 2001.

30 Palley 2011. 
gemeinsamen Währung Euro einer Verschuldung in Fremdwährung gleichkommt. ${ }^{31}$ Der Finanzpolitik ginge jene Handlungsalternative verloren, die alle währungspolitisch souveränen Staaten besitzen, nämlich die Finanzierung über Seigniorage. Vielmehr: Bei Konvergenz der Inflationsraten der Mitgliedsländer kann sich sogar der Spielraum für Regierungen verringern, ihre Budgets wie bisher aus Seigniorage zu finanzieren. Es bleiben als Alternativen für ausgeglichene Budgets nur noch Steuererhöhungen und Ausgabenkürzungen, bei denen die Wahl des Instruments von den jeweiligen marginalen fiskalischen Kosten abhängt: Diese Wahl kann mit Blick auf Einkommen und Beschäftigung auch suboptimal wirken. Längerfristig mag dadurch die Notwendigkeit für Reformen des Steuersystems entstehen. Kurzfristig - also in Krisenzeiten - wirkt eine derartige Einschränkung der Optionen allerdings schädlich, weil eine funktionale Finanzpolitik über fiskalische Defizite zur Stabilisierung von Einkommen und Nachfrage beizutragen hätte.

\section{Die ökonomischen Konsequenzen des Fiskalpakts}

\subsection{Ausgangslage}

Die Verabschiedung des EU-Fiskalpakts im März 2012 32 in Verbindung mit einer Reform des präventiven und korrektiven Arms des Stabilitäts- und Wachstumspakts $^{33}$ wird als wesentlicher Schritt zur Überwindung der Euro-Krise angesehen. Seine geplante Umsetzung ab 2013 trifft auf eine krisenhafte Situation im EuroRaum, die durch eine Spaltung in Gläubiger- und Schuldnerstaaten und eine Rezession in den meisten Mitgliedsländern charakterisiert ist. Abbildung 1 verdeutlicht diese Spaltung seit Beginn der globalen Finanzkrise anhand der Umlaufrenditen zehnjähriger Staatstitel für zwölf Euro-Länder im Vergleich zum längerfristigen Trend der EWU-Durchschnittsrendite. Der Trend (gefettete Kurve) zeigt auch 2012 trotz fiskalischer Anpassungsprogramme keinen Rückgang oder Anstieg gegenüber den seit 2002 erreichten Werten von etwa vier Prozent. Unterhalb des Trends finden sich in der Krise die Umlaufrenditen für sechs Länder (Österreich, Deutschland, Belgien, Frankreich, Finnland und die Niederlande). Unterdurchschnittliche Renditen auf den Sekundärmärkten schlagen sich über den Rückgang der Nominalverzinsung auch auf dem Primärmarkt für Neuemissionen nieder. Abbildung 1 illustriert also eine Umverteilung der Seigniorage zwischen Schuldner- und Gläubigerländern. Für Deutschland habe ich den krisenspezifischen Zinsgewinn auf ca. 50 bis 70 Mrd. Euro geschätzt, unter der Annahme einer Neuemission sechsjähriger Staatstitel von etwa 600 Mrd. Euro im Jahre 2011.34

31 Diese mittlerweile zum Allgemeingut gehörende Erkenntnis scheint zum ersten Mal Sievert 1993 zum Ausdruck gebracht zu haben.

32 European Council 2012 a.

33 European Commission 2012 c.

34 Siehe dazu meinen Beitrag "Warum Deutschland seine Krisengewinne wieder in den Euroraum zurückfließen lassen muss «. http://www.hubert-gabrisch.com (comments and viewpoints) (Zugriff vom 14.01.2013). 
Am aktuellen Rand ähnelt das Bild dem vor Einführung der Gemeinschaftswährung, als Abwertungsrisiken für einzelne Länder deren Risikoaufschläge nach oben trieben. Das reflektierte aber genau jene Alternative, die jede Regierung damals noch besaß, wenn sie sich in eigener Währung verschuldete: Höhere Zinsen auf Staatsanleihen wurden über die Zinseinnahmen der Zentralbank wieder in den Staatshaushalt zurückgeführt. In der gegenwärtigen Lage besteht diese Möglichkeit nicht mehr. Die Regierung eines Schuldnerlandes muss entweder die Steuereinnahmen erhöhen oder die nachfragewirksamen Staatsausgaben senken - beides mit Folgen für die Realwirtschaft.

\section{Abbildung 1: Quartalsweise Umlaufrenditen zehnjähriger Schatztitel in Prozent seit 1990}

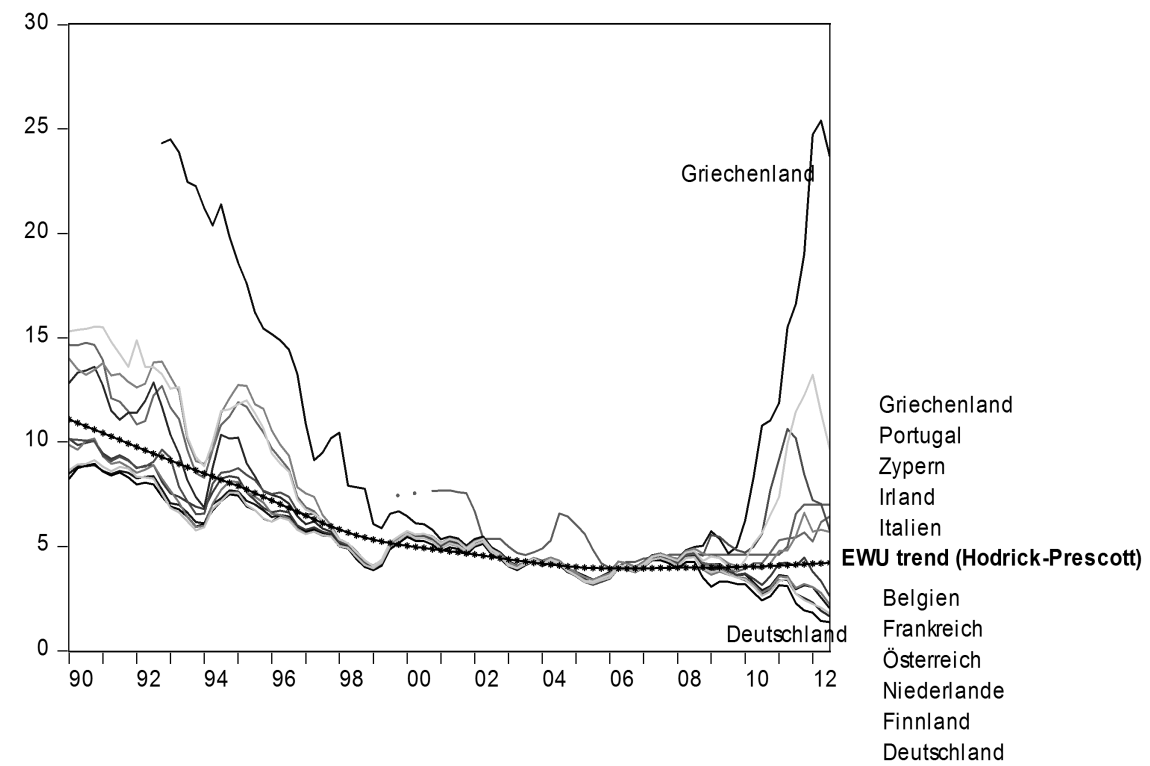

Quelle: European Commission 2012 b (Eurostat), Zinssätze nach Konvergenzkriterium; eigene Darstellung.

Dieser fiskalische Anpassungsprozess hat bereits 2010/2011 in der gesamten EU begonnen und 2012 kontraktive Wirkungen im Euro-Raum ausgelöst. Nach den Zahlen des statistischen Amtes der EU (Eurostat) ${ }^{35}$ ist die aggregierte Wirtschaftsleistung im Euro-Raum - das reale Bruttoinlandsprodukt (BIP) - im zweiten Quartal 2012 um 0,2 Prozent gegenüber dem ersten Quartal gesunken. Verglichen mit dem zweiten Quartal 2011 fiel der Rückgang mit 0,5 Prozent sogar noch höher aus. Ein genauerer Blick enthüllt die eigentlich dramatische Entwicklung: Schuldnerländer, 
die hohe Zinslasten zu tragen haben, leiden stärker unter den sich verschlechternden wirtschaftlichen Aussichten, was es ihnen erschwert, die geforderten fiskalischen Überschüsse zu erreichen, den öffentlichen Schuldenstand zu reduzieren und auch langfristig tragfähige Schuldenstände im privaten Sektor zu sichern. Die Folge: Rating-Agenturen sehen keinen Grund, ihre Risikobewertung zu verbessern, sodass die Zinsbelastung der Staatshaushalte nicht sinkt. Sowohl der öffentliche wie auch der private Sektor in den Schuldnerländern drohen in eine Ponzi-Finanzierung zu rutschen, bei der die Einnahmen für die Bedienung der aufgenommenen Kredite nicht mehr ausreichen, neuer Kredit nicht erreichbar ist und deshalb Vermögensbestände zu gesunkenen Marktpreisen aufgelöst werden müssen. Als Resultat öffentlicher und privater Vermögens- und Schuldenreduzierung droht eine Verschlechterung der Finanzierungsbedingungen für realwirtschaftliche Investitionen in den Schuldnerländern, während in den Gläubigerländern die Kapitalrückflüsse zu einer Verbesserung der Finanzierungsbedingungen führen. Auch dadurch dürfte es zu einer Verstärkung der Divergenz der Wirtschaftsleistung zwischen den EuroMitgliedsländern kommen. Unter diesen Umständen - Rezession, steigende Arbeitslosigkeit, geringer Inflationsdruck - würde eine funktionale Finanzpolitik nicht einen Abbau, sondern einen Anstieg des aggregierten Budgetdefizits fordern (siehe dazu den methodischen Anhang).

\subsection{Auswirkungen auf öffentliche Haushalte und Wirtschaftsleistung}

Der von allen EU-Ländern mit Ausnahme Großbritanniens und Tschechiens ratifizierte Fiskalpakt verlangt stattdessen in der gegenwärtigen Krise eine Reduzierung der Neuverschuldung, also des Budgetdefizits, und darüber hinaus auch des Schuldenstands, was faktisch auf Budgetüberschüsse hinausläuft. Hier seine beiden wichtigsten Vorschriften: ${ }^{36}$ Erstens hat der gesamtstaatliche Haushalt einer Vertragspartei ausgeglichen zu sein oder einen Überschuss auszuweisen. Diese Regel gilt als eingehalten, wenn der jährliche Saldo des strukturellen Haushalts kein höheres Defizit als 0,5 Prozent des Bruttoinlandsprodukts aufweist. Für Mitglieder des EuroRaums gilt, dass das strukturelle Defizit niedriger ausfallen und im Extremfall sogar zu einem Überschuss werden muss, wenn der Stand der öffentlichen Verschuldung oberhalb der 60-Prozent-Grenze des Stabilitäts- und Wachstumspakts liegt. Wie die Anpassung des Budgets für die einzelnen Euro-Länder und den Euro-Raum insgesamt konkret ausfallen soll, obliegt den Berechnungen der Kommission und ihren Verhandlungen mit jedem Land. Dabei sind »außerordentliche Umstände « des Landes zu berücksichtigen, beispielsweise eine schwere Rezession. Zweitens ist eine etwaige Differenz zwischen der Verschuldungsobergrenze von 60 Prozent und einem höheren Verschuldungsstand jährlich um ein Zwanzigstel der jeweils aktuellen Differenz zu verringern. Dies suggeriert auf den ersten Blick eine abnehmende Last der Schuldenreduktion über die Jahre hinweg. Auf den zweiten Blick kann dies nur gelten bei Konstanz der Wirtschaftsleistung. Ein im Zuge der Budgetkürzungen

36 European Council 2012 a.

Leviathan, 41. Jg., 1/2013 
zu erwartender Rückgang der Wirtschaftsleistung würde jedoch den degressiven Effekt abschwächen.

Nachfolgend gibt Tabelle 1 einen Überblick über die voraussichtlichen Budgetanpassungen für die einzelnen Länder und den Euro-Raum insgesamt. Die erste Spalte führt die tatsächlichen fiskalischen Defizite eines jeden Landes und der gesamten Währungsunion auf und folgt der Vorausschau der Europäischen Kommission vom Herbst 2011 (Zypern, Malta und Luxemburg wurden ausgelassen). Die zweite Spalte zeigt die strukturellen Defizite, wie sie von der Kommission ermittelt wurden. Die Differenz zwischen beiden Spalten sind die zyklischen Defizite und sonstigen Haushaltskomponenten. Für den gesamten Währungsraum berechnete die Kommission für 2012 einen Anstieg des konjunkturellen Defizits auf 1,2 Prozent des BIP, das heißt der Euro-Raum befand sich bereits in einem Abschwung. Die dritte Spalte enthält die öffentlichen Schuldenstände in Prozent des BIP im Jahre 2012. Spalte vier weist auf die Lücke zwischen dem 60-Prozent-Kriterium des Stabilitäts- und Wachstumspakts und dem Schuldenstand des Jahres 2012 hin. Leere Zellen besagen, dass der öffentliche Schuldenstand unterhalb des Referenzwerts liegt. Die fünfte Spalte berichtet über die Budgetkürzungen in einem Konsolidierungszeitraum von fünf ${ }^{37}$ Jahren auf Basis des Defizits im strukturellen Haushalt des Jahres 2012. Die sechste Spalte führt die Kürzung des Schuldenstands gemäß der zweiten Vorschrift auf, und zwar ebenfalls für die ersten fünf Jahre.

Ein derartig rigides Paket fiskalischer Kürzungen wird nicht ohne negative Auswirkungen auf Produktion und Beschäftigung bleiben. Die Effekte für die Produktion können mit einem Modell abgeschätzt werden, dessen Grundlage der Einkommensmultiplikator ${ }^{38}$ ist (zu Methode und Daten siehe den Anhang). ${ }^{39}$ Die Durchsetzung der ersten Vorschrift des Fiskalpakts in einem mittelfristigen Anpassungszeitraum von fünf Jahren (2013 bis 2017) dürfte zu einer Reduktion des realen Bruttoinlandsprodukts der Euro-Zone bis zum Jahre 2017 um insgesamt ca. 2,6 Prozent gegenüber dem Ausgangsjahr 2012 führen. Einen nachfrageseitig noch größeren Entzugseffekt könnte allerdings die Anwendung der zweiten Vorschrift verursachen. Bezogen auf die ersten fünf Jahre des langfristigen Anpassungszeitraums, dürfte die degressive Reduktion des Schuldenstands die aggregierte Wirtschaftsleistung so weit reduzieren, dass sie im Jahre 2017 um ca. elf Prozent unter der des Jahres 2012 liegen würde. Eine Formulierung in der ersten Vorschrift (siehe oben) lässt vermuten, dass in allen Ländern mit Ausnahme der Niederlande sogar ein

37 Die Spezifikationen für den korrektiven Arm des Stabilitäts- und Wachstumspakts enthalten eine dreijährige Anpassungsperiode (European Commission 2012 c); ich nehme also hier bereits eine Verlängerung wegen der »außergewöhnlichen Umstände « an.

38 Die häufig in der Literatur angeführten positiven Angebotseffekte einer fiskalischen Konsolidierung, die sogenannten nicht-keynesianischen Effekte, sind bisher nicht aufgetreten und dürften auch mittelfristig keine besondere Bedeutung spielen, sodass hier lediglich die keynesianischen Effekte berücksichtigt werden.

39 Der Leser möge notieren, dass es sich hier um keine Prognose handelt, sondern um die Abschätzung der Entzugseffekte ausschließlich durch die fiskalische Konsolidierung bei Konstanz aller anderen Faktoren. 
Tabelle 1: Auswirkungen des Fiskalpakts auf die gesamtstaatlichen Haushalte der EWU-Länder

\begin{tabular}{|c|c|c|c|c|c|c|}
\hline & \multicolumn{2}{|c|}{$\begin{array}{l}\text { Budgetdefizit (Finanz- } \\
\text { überschuss }(+) / \\
\text {-defizit (-) in Prozent } \\
\text { des BIP für 2012) }\end{array}$} & \multicolumn{2}{|c|}{ Schuldenstand } & \multirow[t]{2}{*}{$\begin{array}{l}\text { Kürzung des struk- } \\
\text { turellen Defizits auf } \\
0,5 \text { Prozent des BIP } \\
\text { in } 5 \text { Jahren }^{\mathrm{a}}\end{array}$} & \multirow{2}{*}{$\begin{array}{l}\text { Maximale Kür- } \\
\text { zung des Schul- } \\
\text { denstands in } \\
\text { Prozent des BIP } \\
\text { in einem Zeit- } \\
\text { raum von } 5 \\
\text { Jahren }\end{array}$} \\
\hline & $\begin{array}{l}\text { Tatsäch- } \\
\text { lich }\end{array}$ & $\begin{array}{l}\text { Struktu- } \\
\text { relles } \\
\text { Defizit }\end{array}$ & $\begin{array}{l}2012 \text { in } \\
\text { Prozent } \\
\text { des BIP }\end{array}$ & $\begin{array}{l}\text { Überstei- } \\
\text { gen der } \\
60 \text {-Pro- } \\
\text { zent- } \\
\text { Schwelle }\end{array}$ & & \\
\hline $\begin{array}{l}\text { Deutsch- } \\
\text { land }\end{array}$ & $-1,0$ & $-0,7$ & 81,2 & 21,0 & 0,2 & 4,8 \\
\hline Belgien & $-4,6$ & $-4,0$ & 99,0 & 39,0 & $3,5(0,7)$ & 8,8 \\
\hline Estland & $-1,8$ & $-1,7$ & 6,0 & & $1,2(0,2)$ & \\
\hline Irland & $-8,6$ & $-8,0$ & 117,5 & 57,5 & $7,5(1,5)$ & 13,0 \\
\hline $\begin{array}{l}\text { Griechen- } \\
\text { land }\end{array}$ & $-7,0$ & $-2,9$ & $160,0^{\mathrm{b}}$ & 100,0 & $2,4(0,5)$ & 22,6 \\
\hline Spanien & $-5,9$ & $-4,2$ & 73,8 & 13,8 & $3,7(0,7)$ & 3,1 \\
\hline Frankreich & $-5,3$ & $-4,0$ & 89,2 & 29,2 & $3,5(0,7)$ & 6,6 \\
\hline Italien & $-2,3$ & $-1,3$ & 120,5 & 60,5 & $0,8(0,2)$ & 13,7 \\
\hline $\begin{array}{l}\text { Nieder- } \\
\text { lande }\end{array}$ & $-3,1$ & $-1,8$ & 64,9 & 4,9 & $1,3(0,9)$ & 1,1 \\
\hline Österreich & $-3,1$ & $-2,7$ & 73,3 & 13,3 & $2,2(0,4)$ & 3,1 \\
\hline Portugal & $-4,5$ & $-2,4$ & 111,0 & 51,0 & $1,9(0,4)$ & 11,5 \\
\hline Slowenien & $-5,3$ & $-3,8$ & 50,1 & & $3,3(0,7)$ & \\
\hline Slowakei & $-4,9$ & $-4,4$ & 47,5 & & $3,9(0,8)$ & \\
\hline Finnland & $-0,7$ & 0,3 & 51,8 & & 0,0 & \\
\hline EWU-17 & $-3,4$ & $-2,0$ & 90,4 & 30,4 & $1,5(0,3)$ & 6,9 \\
\hline
\end{tabular}

a jabresdurchschnittliche Werte in Klammern

${ }^{b}$ nach dem Schuldenschnitt im Rettungspaket für Griechenland

Quellen: European Commission 2012 a für die Verschuldungsdaten beziehungsweise European Commission 2012 b für die Budgetdaten (Ameco Datenbank); eigene Berechnungen.

Überschuss im strukturellen Haushalt erzielt werden muss. Dies würde dann auch die Erfüllung der ersten Vorschrift sicherstellen. 
Tabelle 2: Schätzung der Auswirkungen des Fiskalpakts auf die öffentlichen Haushalte und die Wirtschaftsleistunga im Euro-Raum

\begin{tabular}{|c|c|c|c|c|c|c|}
\hline & $2012^{a}$ & 2013 & 2014 & 2015 & 2016 & 2017 \\
\hline \multicolumn{7}{|l|}{ Anpassung gemäß der ersten Vorschrift des Fiskalpakts } \\
\hline $\begin{array}{l}\text { Finanzierungsdefizit (-) bzw. - überschuss }(+)^{\mathrm{b}} \\
\text { Gesamtstaat ohne Zinszahlungen in Mrd. Euro }\end{array}$ & $-0,4$ & 28 & 57 & 87 & 115 & 144 \\
\hline $\begin{array}{l}\text { Defizit (-) im strukturellen Haushalt in Prozent des } \\
\text { BIPa }\end{array}$ & $-2,0$ & $-1,7$ & $-1,4$ & $-1,1$ & $-0,8$ & $-0,5$ \\
\hline BIP zu Marktpreisen 2012 in Mrd. Euro & 9.538 & 9.489 & 9.440 & 9.391 & 9.342 & 9.294 \\
\hline Veränderungsrate des BIP in Prozent & 1,2 & $-0,51$ & $-0,52$ & $-0,52$ & $-0,52$ & $-0,52$ \\
\hline \multicolumn{7}{|c|}{ Anpassung gemäß der zweiten Vorschrift des Fiskalpakts } \\
\hline $\begin{array}{l}\text { Reduktion des Schuldenstands (degressiv) in Pro- } \\
\text { zent des BIPa }\end{array}$ & & $-1,5$ & $-1,4$ & $-1,4$ & $-1,3$ & $-1,3$ \\
\hline $\begin{array}{l}\text { Finanzierungssaldo Gesamtstaat ohne Zinszahlun- } \\
\text { gen in Mrd. Euro }\end{array}$ & $-0,4$ & 145 & 279 & 403 & 519 & 626 \\
\hline BIP zu Marktpreisen 2012 in Mrd. Euro & 9.538 & 9.292 & 9.069 & 8.867 & 8.682 & 8.513 \\
\hline Veränderungsrate des BIP in Prozent & 1,2 & $-2,6$ & $-2,4$ & $-2,2$ & $-2,1$ & $-1,9$ \\
\hline
\end{tabular}

a 2012: Zablen der Kommission

${ }^{b}$ Summe aus Saldo struktureller Haushalt, konjunktureller Haushalt plus sonstiger Positionen.

Quelle: European Commission 2012 b; Berechnungen des Autors (siehe auch Anhang).

Die durch den Fiskalpakt ausgelöste Rezession fiele regional natürlich unterschiedlich aus. Damit bleibt zu befürchten, dass sich die bestehende Spaltung zwischen Gläubiger- und Schuldnerstaaten vertiefen wird, was sowohl die realwirtschaftliche Entwicklung betrifft wie auch die Risikoaufschläge auf Staatsanleihen.

\section{Zur Überwindung der Euro-Krise}

Die Ausführungen zielten darauf ab, das Verständnis für die Schaffung von Institutionen der $\mathrm{E}(\mathrm{W}) \mathrm{U}$ zu wecken, die eine Geld- und Fiskalpolitik aus einer gesamtwirtschaftlichen Perspektive erlauben. Der Notwendigkeit, aus einem Halbwaisen einen souveränen Euro zu machen, verlangt nach Möglichkeiten für eine funktionale Finanzpolitik auf dem Gebiet der gemeinsamen Währung, die mit der Geldpolitik der EZB abgestimmt werden kann. Auf Ebene der Mitgliedstaaten ist dies nicht mehr möglich. Der praktisch unkündbare Fiskalpakt erlaubt es derzeit nicht, über eine koordinierte Defizitausweitung die Beschäftigung im Euro-Raum zu sichern und die Wirtschaftsleistung zumindest aufrechtzuerhalten. Kontroll- und Sanktionsmechanismen für die regionale Ebene eines Wirtschaftsraums werden aber erst dann ökonomisch sinnvoll, wenn sie auf zentraler Ebene mit einer funktionalen Wirtschaftspolitik kombiniert werden können, so wie dies in souveränen Währungsräumen der Fall ist wie etwa in den USA (Insolvenzordnung für Bundesstaaten) oder Deutschland vor Einführung der Schuldenbremse (Bundeszwang bei einem finanziellen Notstand eines Bundeslandes). 
Dem Fiskalpakt fehlt also ein Überbau. In einer idealisierten Form besteht dieser Überbau in der Schaffung einer zentralen fiskalischen Kapazität, sei es als europäisches Schatzamt oder Finanzministerium, das anstelle der bisher 17 nationalen Finanzminister alleiniger Partner der EZB im Krisenmanagement wäre. Wie George Soros treffend feststellt: ${ }^{40}$ Eine EU-weite Finanzbehörde sei »die fehlende Zutat, um den Euro zu einer vollwertigen Währung mit einem echten Kreditgeber der letzten Instanz zu machen «. Dazu müssten allerdings jene Funktionen von der nationalen auf die europäische Ebene übertragen werden, die für einen souveränen Euro lebensnotwendig sind. Die Ausführung der Funktionen wäre auch auf der europäischen Ebene demokratisch zu legitimieren, etwa über erweiterte Rechte des EU-Parlaments. Im Zentrum stehen drei Funktionen der Finanzbehörde: (a) Verhinderung wirtschaftlicher Ungleichgewichte im Verhältnis der Mitgliedsländer untereinander, (b) Sicherung eines einheitlichen Marktes für Staatsanleihen in Euro und (c), ja, die Aufsicht über die Einhaltung des Fiskalpakts und seine Durchsetzung.

Neben horizontalen Transfers können die Anpassungslasten aus Ungleichgewichten zwischen den Teilnehmerländern an der Währungsunion auch über vertikale Transfers gemildert werden. Sie würden die Nachfrage stabilisieren und Investitionsanreize liefern, die scharfe und rapide erfolgende Aufschwünge und Kontraktionen auf den Finanzmärkten abmildern, wenn nicht gar verhindern - so wie dies beispielsweise im Verhältnis der Regionen in Deutschland der Fall ist: Regionale $\mathrm{Zu}$ - und Abflüsse von Kapital finden zwar statt, stellen aber kein Problem für die Gesamtwirtschaft dar, weil Ausgleichsmechanismen Übertreibungen der Finanzmärkte verhindern. Vorschläge für die Einrichtung eines derartigen Transfersystems sind seit 1993 immer wieder im Gespräch gewesen, auch aus der EU-Kommission heraus. ${ }^{41}$ Der Vorschlag einer Studie des Jacques-Delors-Institute ${ }^{42}$ geht aktuell am weitesten: Die nationalen Haushalte sollten in Zeiten eines wirtschaftlichen Aufschwungs Beiträge an einen Fonds der Euro-Länder abführen, aus dem im Falle eines Abschwungs Zahlungen an nationale Haushalte zu leisten wären. Funktionale Finanzpolitik setzt allerdings voraus, dass die Beitragssätze und Regeln bei außergewöhnlichen Erfordernissen geändert werden können.

Bei ungewöhnlich starken und lang anhaltenden Schocks, wie sie besonders auf den globalisierten Finanzmärkten verursacht werden, ist die Stabilität der Finanzmärkte von der Existenz risikoarmer Wertpapiere abhängig, die in der Regel von der öffentlichen Hand emittiert werden. Asymmetrische Zinsschocks können in einem Währungsraum nur dann verhindert werden, wenn ein einheitlicher Markt für risikoarme Wertpapiere besteht, ansonsten würden regional unterschiedliche Zinsniveaus nicht nur die Anlageattraktivität der Währung insgesamt schwächen, sondern auch zu regional unterschiedlichen realwirtschaftlichen Effekten führen. Die Sicherung eines einheitlichen Marktes für Staatspapiere setzt eine gemeinschaftliche, durchaus konditionierte Haftung für jenen Teil der öffentlichen Verschuldung

40 Soros 2012, S. 10.

41 SMR 1993; von Hagen, Hammond 1998; zur Schaffung einer europaweiten Arbeitslosenversicherung vgl. Dullien 2008.

42 Notre Europe Jacques Delors Institute 2012, S. 30 f.

Leviathan, 41. Jg., 1/2013 
eines Landes voraus, der eine vorher vereinbarte Schwelle überschreitet, wie es auch einige Vorschläge vorsehen. ${ }^{43}$ Wie auch immer dies technisch im Einzelnen gestaltet wird: Die gemeinschaftliche Haftung kann letztlich nur aus der aggregierten Steuerkraft der Teilnehmerländer stammen, selbst wenn die Herausgabe von EuroBonds zunächst über Kreditaufnahme an den Finanzmärkten oder von der EZB finanziert werden soll. Ein europäisches Schatzamt müsste deshalb, um ausreichend »Feuerkraft « in Abstimmung mit einer LOLR-Funktion der EZB zu erzielen, im Bedarfsfalle auch das Recht auf eigene Einnahmen besitzen, die entweder aus einer eigenen europäischen Steuer stammen oder aus Abführungen aus den nationalen Steuereinnahmen.

\section{Abschließende Bemerkungen}

In diesem Beitrag habe ich argumentiert, dass das Fehlen eines europäischen Souveräns ein grundlegendes Problem der europäischen Gemeinschaftswährung ist, aus dem sich andere Probleme ableiten, zum Beispiel nicht tragfähige Schuldner- und Gläubigerpositionen (= Leistungsbilanzungleichgewichte) der Mitgliedsländer. Die Finanzmärkte benötigten ca. elf Jahre, um dies herauszufinden - mit der Folge einer regionalen Aufsplitterung des einheitlichen Euro-Marktes für Staatspapiere in Gläubiger- und Schuldnermärkte und riesiger Risiken für beide Seiten. Kleine regionale Euro-Märkte sind jedoch wenig attraktiv für Großanleger. Die Ausführungen haben ferner demonstrieren sollen, dass die Theorie des optimalen Währungsraums kein guter Leitfaden in der Reformdebatte ist, da sie die Rolle des Staates ausklammert und zu viel Wert auf die Reform von Märkten legt. Das Ziel, den Euro zu einer souveränen Währung werden zu lassen, können Reformvorschläge, die auf marktfundamentalistischen Überzeugungen fußen, nicht erreichen. Der Fiskalpakt, von dem gesagt wird, er sei der erste Schritt in eine Fiskalunion, zielt jedoch darauf ab, die letzten Spielräume für eine funktionale Finanzpolitik zu beseitigen. Eine der wahrscheinlichen Konsequenzen besteht in der Strangulierung der europäischen Wirtschaft und in einer weiteren Schwächung des Euro als Reservewährung.

Der Gedanke, eine zentrale »fiskalische Kapazität « zum Kern einer Reform der $\mathrm{E}(\mathrm{W}) \mathrm{U}-\mathrm{Institutionen}$ zu machen, ist mittlerweile weit verbreitet. Gerade jene, die skeptisch gegenüber der Reformfähigkeit der EU eingestellt sind, mag der Vorschlag einer Gruppe um den EU-Ratspräsidenten Herman Van Rompuy überraschen: ${ }^{44}$ »Eine voll entfaltete Fiskalunion impliziert die Entwicklung einer größeren Kompetenz auf europäischer Ebene mit der Fähigkeit, die gegenseitigen ökonomischen Abhängigkeiten zu beherrschen, und würde letztlich auch die Entwicklung einer Fiskalbehörde auf europäischer Ebene mit sich bringen, eine Art europäisches Finanzministerium. Dabei müssten die entsprechende Rolle und die Funktionen eines gesamteuropäischen Haushaltsbudgets sowie die Rolle der nationalen Haus-

43 »European safe bonds " gemäß Brunnermeier et al. 2011; aber auch INET 2012; Notre Europe Jacques Delors Institute 2012.

44 European Council 2012 b, S. 6. 
halte definiert werden «. Es bleibt abzuwarten, welche Chance auf Verwirklichung Statements wie das obige des EU-Ratspräsidenten tatsächlich hat.

\section{Anhang: Methodik und Daten der BIP-Schätzungen}

\section{Funktionale Finanzpolitik}

Als analytischen Rahmen verwende ich die folgende Gleichung für eine monetäre Ökonomie, in der die Ausgaben die Einkommen und damit auch die Ersparnisse aus den Einkommen schaffen:

$$
Y^{d}+T+M=C+I+G+X
$$

Die linke Seite der Gleichung ist die Einkommensseite, bestehend aus dem verfügbaren Einkommen $Y^{\mathrm{d}}$ des privaten Sektors, dem verfügbaren Einkommen der Regierung $T$ und den verfügbaren Einkommen des Auslands aus den Importen $M$. Die Ausgaben auf der rechten Seite umfassen den privaten Konsum C, die Investitionen des privaten Sektors $I$, die Ausgaben der Regierung für Güter, Dienstleistungen und öffentliche Investitionen $G$ und die Ausgaben des Auslands für Exporte (Güter des Inlands) $X$. Eine Umstellung von (1) führt zu

$$
N P S=D+E
$$

wobei NPS $=S \mathrm{p}-I$ beziehungsweise die Nettoersparnisse des privaten Sektors. $S \mathrm{p}$ $=Y^{\mathrm{d}}-\mathrm{C}$ sind die privaten Ersparnisse (private Haushalte und nicht verteilte Gewinne des Unternehmenssektors), $I$ die privaten Investitionen, und $D$ ist das primäre Budgetdefizit der Regierung mit $D=G-T ;{ }^{45}$ außerdem gilt für die Nettoexporte $E=X-M$.

Angenommen, E sei 0: Dann würden die privaten Nettoersparnisse dem Budgetdefizit entsprechen. Wenn unter diesen Umständen die Nettoersparnisse positiv wären, dann muss auch das Budgetdefizit $D$ größer als Null sein. Wenn also die wirtschaftliche Lage generell durch Schuldenabbau bei privaten Haushalten, Unternehmen und Banken gekennzeichnet wäre, weil sie ihre eigene Bilanz durch Ausgabenkürzungen bei Konsum, Investitionen und einem Anstieg der Ersparnis zu konsolidieren versuchten, so wie dies gegenwärtig im Euro-Raum zu beobachten ist, gilt grundsätzlich: $\triangle N P S>0$, was notwendigerweise $\triangle(D+E)>0$ erfordert. Angenommen, die Nettoexporte seien sehr gering, gemessen am BIP - eine Annahme, die für den Euro-Raum zutrifft -, dann würde ihr Anstieg nicht sehr viel für die Stabilisierung von Produktion, Beschäftigung und Einkommen bringen. Es bleibt also nur die Erhöhung des Haushaltsdefizits im gesamten Euro-Raum und nicht seine Reduzierung, wie im Fiskalpakt gefordert.

\section{Der Budgetmultiplikator}

Im Unterschied zu den üblichen Ausgaben- oder Einnahmenmultiplikatoren wird hier ein Budgetmultiplikator verwendet, denn die Vorschrift des Fiskalpakts ist die

45 Primärdefizit: Einnahmen und Ausgaben ohne Zinszahlungen.

Leviathan, 41. Jg., 1/2013 
Reduzierung des Budgetdefizits und nicht die Kürzung von Ausgaben oder die Erhöhung von Einnahmen. In der Praxis ist ein Mix von beidem zu erwarten. Der Ausgabenmultiplikator ist generell höher als der Einnahmenmultiplikator; der Budgetmultiplikator dürfte in der Mitte zwischen beiden liegen. Eine Umformulierung von (1) beziehungsweise (2) führt zu

$$
S^{p}=D+I+(X-M)
$$

wobei $S \mathrm{p}$ und $M$ Funktionen des verfügbaren Einkommens $Y^{\mathrm{d}}$ sind. Dann gilt $(s+m) Y^{d}=D+I+X$

Auf der linken Seite steht $s$ für die Sparquote oder auch »Sparneigung « der Privaten und $m$ für die Importquote der Volkswirtschaft (»Importneigung «). Auf der rechten Seite sind die Investitionen der Unternehmen und die Exporte exogen, das heißt sie werden nicht durch das inländische Einkommen determiniert. ${ }^{46}$ Der Multiplikator lautet dann:

$$
\Delta Y^{d}=\frac{1}{s+m} \Delta D
$$

beziehungsweise

$$
g=\frac{\Delta Y^{d}}{Y_{t}^{d}}=\frac{1}{s+m}\left(\frac{\Delta D}{Y_{t}^{d}}\right)
$$

mit $g$ als realer Veränderungsrate, wenn beide Seiten durch das Einkommen im Ausgangsjahr $t$ dividiert werden. 1/(s+m) ist der Budgetmultiplikator. Wie ersichtlich, enthält dieser nicht wie üblich einen Steuersatz; dieser steckt in D und kann daher bei einem Budgetmultiplikator nicht zweimal in der Gleichung erscheinen. Das Budgetdefizit $D$ setzt sich aus einem einkommensabhängigen Teil (konjunkturelles Defizit) $D^{\mathrm{k}}$ und einem vom Einkommen unabhängigen Teil (strukturelles Defizit) $\bar{D}^{s}$ zusammen. Der Strich über dem strukturellen Defizit symbolisiert, dass es sich um eine Variable mit politisch vorgegebenem Wert wie im Fiskalpakt handeln soll. Die für die Schätzungen verwendete Multiplikatorgleichung lautet dann

$$
g=\frac{\Delta Y^{d}}{Y_{t}^{d}}=\frac{1}{s+m}\left(\frac{\Delta\left(D^{k}+\overline{D^{s}}\right.}{Y_{t}^{d}}\right)
$$

\section{Daten und Annahmen}

Meine Schätzung der Effekte für den Zeitraum 2013-2017 verwendet nominale Daten der Volkswirtschaftlichen Gesamtrechnung und die Prognose der EU-Kommission für 2012 vom Frühjahr desselben Jahres (siehe Tabelle 3). Angaben in Euro für den Schätzzeitraum sind zu Preisen des Jahres 2012, reflektieren also annähernd reale Größen. Der Primärhaushalt wird als »Finanzierungssaldo Gesamtstaat»

46 Die Exporte werden durch das Einkommen des Auslands bestimmt, die privaten Investitionen folgen den animal spirits der Unternehmer. 
bezeichnet. Der strukturelle Haushalt wies 2012 ein Defizit D in Höhe von zwei Prozent des Bruttoinlandsprodukts zu Marktpreisen aus. Hinzu kamen das Defizit im zyklischen Haushalt sowie übrige Komponenten des Finanzierungssaldos mit einem Überschuss.

Wie erwartet, stellten die Nettoexporte mit durchschnittlich 1,4 Prozent zwischen 2004 und 2012 einen nur geringen Anteil am BIP des Euro-Raums. Die private Ersparnis wurde entsprechend den Gleichungen (1) und (2) aus drei Größen ermittelt: primärer Finanzierungssaldo, private Investitionen und Nettoexporte. Da Sparund Importneigung über die Jahre hinweg volatil waren, wurden für die Schätzung durchschnittliche Werte des Zeitraums 2004 bis 2012 verwendet. Bei der Sparquote ergab sich ein Wert von 0,198. Die für diesen Zeitraum durchschnittliche Importquote belief sich auf 0,392. Bei den Importen wird nicht zwischen Importen für den Export und für den inländischen Verbrauch unterschieden, sodass die berechnete Importneigung etwas überhöht und der Multiplikator etwas zu niedrig erscheint. Der durchschnittliche Budgetmultiplikator nimmt für die Schätzperiode einen Wert von knapp unter 1,7 ein $(1 /(0,198+0,392)$. Vor dem Hintergrund einiger aktueller Schätzungen von Ausgabenmultiplikatoren in Rezessionszeiten - zu erwähnen ist insbesondere eine neuere Schätzung des IWF ${ }^{47}$ - erscheint dieser Wert nicht überhöht. Für die Schätzung wurden konstante zyklische (und übrige Komponenten) des gesamten Finanzhaushalts in Mrd. Euro angenommen, sodass sich ihre Quote am Bruttoinlandsprodukt im Zuge einer rezessiven Entwicklung erhöht. Gleichwohl ist mir bewusst, dass diese Annahme weitere Ungenauigkeiten in der Schätzung verursacht. 


\section{Tabelle 3: Ausgewählte gesamtwirtschaftliche Aggregatea und Kennziffernb für den Euro-Raum (17 Länder)}

\begin{tabular}{|c|c|c|c|c|c|c|c|c|c|}
\hline Aggregat in Mrd. Euro bzw. Kennziffer & 2004 & 2005 & 2006 & 2007 & 2008 & 2009 & 2010 & 2011 & 2012 \\
\hline Bruttoinlandsprodukt zu Marktpreisen $=Y^{\mathrm{d}}$ & 7.860 & 8.145 & 8.564 & 9.030 & 9.242 & 8.922 & 9.176 & 9.421 & 9.538 \\
\hline $\begin{array}{l}\text { Nettoexporte von Gütern und Dienstleistun- } \\
\text { gen }=E\end{array}$ & 159 & 120 & 97 & 131 & 86 & 118 & 123 & 138 & 183 \\
\hline $\begin{array}{l}\text { Importe von Gütern und Dienstleistungen }= \\
M\end{array}$ & 2.728 & 2.985 & 3.362 & 3.619 & 3.799 & 3.168 & 3.647 & 4.011 & 4.169 \\
\hline Bruttoinvestitionen privater Sektor $=I$ & 1.422 & 1.499 & 1.659 & 1.810 & 1.807 & 1.430 & 1.535 & 1.625 & 1.614 \\
\hline Finanzierungssaldo Gesamtstaat ${ }^{\mathrm{c}}=D$ & 15.0 & 36.9 & 131.9 & 206.6 & 84.9 & -314.1 & -313.4 & -100.3 & -0.4 \\
\hline Struktureller Haushalt $=D^{\mathrm{s}}$ & -220 & -196 & -172 & -172 & -268 & -410 & -468 & -311 & -191 \\
\hline Konjunktureller Haushalt $=D^{k}$ & -8 & -8 & 51 & 108 & 65 & -161 & -110 & -75 & -115 \\
\hline Übrige Komponenten $^{\mathrm{b}}=D-\left(D^{\mathrm{s}}+D^{\mathrm{k}}\right)$ & 243 & 241 & 252 & 270 & 288 & 257 & 265 & 286 & 305 \\
\hline Private Ersparnis ${ }^{\mathrm{b}}: S^{\mathrm{pr}}=D+I+E$ & 1.565 & 1.582 & 1.624 & 1.735 & 1.807 & 1.862 & 1.971 & 1.864 & 1.798 \\
\hline Private Sparquote: $s=S^{\mathrm{pr}} / Y$ & 0,199 & 0,194 & 0,190 & 0,192 & 0,196 & 0,209 & 0,215 & 0,198 & 0,188 \\
\hline Importquote: $m=M / Y$ & 0,347 & 0,366 & 0,393 & 0,401 & 0,411 & 0,355 & 0,397 & 0,426 & 0,436 \\
\hline Multiplikator $=1 /(s+m)$ & 1,831 & 1,784 & 1,718 & 1,687 & 1,648 & 1,774 & 1,633 & 1,604 & 1,601 \\
\hline
\end{tabular}

a Zablen der Kommission

${ }^{b}$ Eigene Berechnungen nach Zablen der Kommission

c Primärdefizit: ohne Zinszablungen

Quellen: European Commission (Eurostat) 2012 a; European Commission 2012 b; Berechnungen des Autors.

\section{Literatur}

Baldwin, Richard; Wyplosz, Charles 2009. The economics of European integration. 3. Auflage. New York: McGraw-Hill.

Bayoumi, Tamim; Eichengreen, Barry 1993. "Shocking aspects of European monetary integration ", in Adjustment for growth in the European Monetary Union, hrsg. v. Torres, Francisco; Giavazzi, Francesco, S. 193-229. New York: Cambridge University Press.

Bibow, Jörg 2001. Reflections on the current fashion for central bank independence. Working Paper No. 334. Red Hook: The Jerome Levy Institute of Bard College.

Brunnermeier, Markus K. et al. 2011. European Save Bonds (ESBies). http://euro-nomics.com/ (Zugriff vom 15.07.2012).

Buiter, Willem H. 2008. Can central banks go broke? Discussion Paper No. 6827. Centre for Economic Policy Research.

Dullien, Sebastian 2008. Eine Arbeitslosenversicherung für die Eurozone - Ein Vorschlag zur Stabilisierung divergierender Wirtschaftsentwicklungen in der Europäischen Währungsunion. Berlin: Stiftung Wissenschaft und Politik.

European Commission 2012 a. Ameco databank. General government data. Cyclical adjustment of budget balances. Spring 2012. http://ec.europa.eu/economy_finance/db_indicators/gen_ gov_data/index_en.htm (Zugriff vom 01.10.2012).

European Commission 2012 b. Eurostat. National accounts. http://epp.eurostat.ec.europa.eu/p ortal/page/portal/ (Zugriff vom 04.10.2012).

European Commission 2012 c. Specifications on the implementation of the stability and growth pact and guidelines on the format and content of stability and convergence programmes. 
http://ec.europa.eu/economy_finance/economic_governance/sgp/pdf/coc/2009-11-19_code_ of_conduct_(consolidated)_en.pdf (Zugriff vom 03.09.2012).

European Council 2012 a. Vertrag über Stabilität, Koordinierung und Stenerung in der Wirtschafts- und Währungsunion. http://european-council.europa.eu/media/639244/04_-_tscg. de.12.pdf (Zugriff vom 14.04.2012).

European Council 2012 b. Towards a genuine economic and monetary union. Report by President of the European Council Herman Van Rompuy. http://ec.europa.eu/economy_finance/ focuson/crisis/documents/131201_en.pdf (Zugriff vom 04.10.2012).

Ellis, Joseph J. 2002. Sie schufen Amerika. Die Gründergeneration von John Adams bis George Washington. München: C. H. Beck.

Fontana, Guiseppe 2003. "Post Keynesian approaches to endogenous money: a time framework explanation ", in Review of Political Economy 15, 3, S. 291-314.

Frankel, Jeffrey A.; Rose, Andrew K. 1998. "The endogeneity of the optimum currency area criteria ", in The Economic Journal 108, S. 1009-1025.

Goodhart, Charles E. 1998. "Two concepts of money: implications for the analysis of optimal currency areas ", in European Journal of Political Economy 14, S. 407-432.

Goodhart, Charles E. 1999. "Myths about the lender of last resort ", in International Finance 2, 3, S. 339-360.

Grierson, Philip 1977. The origins of money. Pamphlet. London: Athlone Press (reprinted and revised from Creighton Lecture, Cambridge, 1970).

Grauwe, Paul de 1994. The economics of monetary integration. Second revised edition. Oxford: Oxford University Press.

Hagen, Jürgen von; Hammond, George W. 1998. »Regional insurance against asymmetric shocks. An empirical study for the European Community «. In The Manchester School 66, 3, S. 331-353.

Illing, Gerhard; Jauch, Sebastian; Zabel, Michael 2012. "Die Diskussion um den Euro", in Leviathan 40, 2, S. 156-172.

IMF 2012. World Economic Outlook. http://www.imf.org/external/pubs/ft/weo/2012/02/pdf/c 1.pdf (Zugriff vom 25.10.2012).

INET Council for the Euro Zone Crisis 2011. Breaking the deadlock: a path out of the crisis. September 2011. http://ineteconomics.org/council-euro-zone-crisis/statement (Zugriff vom 20.09.2012).

Kenen, Peter 1969. »The theory of optimum currency areas: an eclectic view ", in Monetary problems in the international economy, hrsg. v. Mundell, Robert A.; Swoboda, Alexander K., S. 41-60. Chicago: University of Chicago Press.

Keynes, John M. 1935. A treatise on money. London: Macmillan.

Knapp, George Friedrich 1924. The state theory of money. London: Macmillan.

Krugman, Paul 1993. "Lessons of Massachusetts for EMU «, in Adjustment for growth in the European Monetary Union, hrsg. v. Torres, Francisco; Giavazzi, Francesco, S. 241-261. New York: Cambridge University Press.

McKinnon, Ronald 1963. "Optimum currency areas", in American Economic Review 53, S. 717-724.

McKinnon, Ronald 2002. „Optimum currency areas and the European experience«, in Economics of Transition 10, 2, S. 343-364.

Lavoie, Marc 2011. The monetary and fiscal nexus of neo-chartalism: a friendly critical look. Mimeo. Department of Economics, University of Ottawa.

Lerner, Abba. P. 1943. »Functional finance and the federal debt ", in Social Research 10 , S. 38-51.

Mundell, Robert A. 1961. "A theory of optimum currency areas ", in American Economic Review 51, 4, S. 657-665.

Mundell, Robert A. 1973. »Uncommon arguments for common currencies «, in The economics of common currencies, hrsg. v. Johnson, Harry G.; Swoboda, Alexander K., S. 114-132. London: Allen \& Unwin.

Notre Europe Jacques Delors Institute 2012. Completing the Euro. A roadmap towards fiscal union in Europe. Paris. http://www.eng.notre-europe.eu/011-14078-Presentation-of-the-re port-of-the-Tommaso-Padoa-Schioppa-group-Paris.html (Zugriff vom 17.10.2012).

Leviathan, 41. Jg., 1/2013 
Palley, Tom 2011. Monetary union stability: the need for a government banker und the case for a European public finance authority. IMK Working Paper 2/2011. Düsseldorf: Hans-Böckler-Stiftung.

Schumpeter, Joseph A. 1994 [1954]. History of economic analysis. London: Routledge.

SMR 1993. Stable money - sound finances. Community public finance in the perspective of EMU. European Economy No. 53. Brüssel: Commission of the European Comunities.

Sievert, Olaf 1993. "Geld, das man nicht selbst herstellen kann - Ein ordnungspolitisches Plädoyer für die Europäische Währungsunion ", in Währungsunion oder Währungschaos? Was kommt nach der D-Mark? , hrsg. v. Bofinger, Peter; Collignon, Stephan; Lipp, Ernst-Moritz, S. 13-24. Wiesbaden: Gabler.

Soros, George 2012. "Deutschland muss führen oder aus dem Euro austreten «, in Spiegel online. http://www.spiegel.de/wirtschaft/george-soros-deutschland-muss-fuehren-oder-aus-dem-eu ro-austreten-a-854595.html (Zugriff vom 09.09.2012).

Wagener, Hans-Jürgen; Eger, Thomas 2009. Europäische Integration. München: Vahlen. 
Zusammenfassung: Ich argumentiere, dass eine Währung einen Souverän braucht, um Stabilität auf den Finanzmärkten und in der Realwirtschaft zu sichern. Andernfalls würde eine Währungsunion über kurz oder lang zerfallen. Insofern ist die aktuelle Krise des Euro-Raums auf das Fehlen eines Souveräns zurückzuführen. Die Theorie des optimalen Währungsraums bringt keine Erkenntnisse zur Überwindung der Krise, weil sie die Separierung von Geld und Staat als Grundlage hat. Auch deshalb liefert sie eher eine Begründung für Reformen wie den Fiskalpakt, dem zufolge fiskalische Operationen von der Einschätzung der Finanzmärkte abhängen sollen. Ich zeige, wie der Fiskalpakt im Gegenteil zu einer tiefen Rezession und zu einer dauerhaften Kluft zwischen Gläubiger- und Schuldnerländern führen wird. Notwendig ist vielmehr eine Transformation der Währungsunion in einen souveränen Währungsraum, in dem eine effektive Koordination von Geld- und Fiskalpolitik zwischen einer EU-Finanzbehörde und der Zentralbank im Sinne einer funktionalen Fiskalpolitik möglich wird.

Stichworte: Euro-Krise, optimale Währungsräume, Geld, funktionale Finanzpolitik, Fiskalpakt, Systemtransformation.

\title{
Currency without a sovereign: on the origin of the Euro crisis and reform attempts
}

Summary: The argumentation is that every currency needs a sovereign authority for stabilizing the financial market and the real economy. Failing this, a currency union will sooner or later decay. Thus the current crisis of the Euro area is the result of the lack of a sovereign. The theory of optimum currency area does not equip us with sufficient knowledge for overcoming the crisis, since it is based on a separation of money and state; it thus provides a reason for reforms such as the fiscal compact, where fiscal operations ought to depend on assessments made by financial markets. As is demonstrated, the implementation of this pact will lead to a deeper crisis of the Euro area and to a deeper split into creditor and debtor countries. What is necessary is that the currency union be transformed into a sovereign currency area in which monetary and fiscal policies are effectively coordinated with a central financial authority and the central bank in the sense of the concept of functional financial policy.

Keywords: Euro crisis, optimum currency area, money, functional finance, fiscal compact, system transformation.

\author{
Autor \\ Dr. Hubert Gabrisch \\ Institut für Wirtschaftsforschung Halle \\ Kleine Märkerstr. 8 \\ 06108 Halle (Saale) \\ gab@iwh-halle.de
}

Leviathan, 41. Jg., 1/2013 